\title{
Investigation of Insulin Sensitivity in Children of Different Ages by Glucose, Insulin, and Somatostatin Infusion
}

\author{
Keisuke Okasora' ${ }^{1}$, Masakuni Tokuda ${ }^{3}$, Kazutaka Konishi ${ }^{4}$, Tatsuya Oguni ${ }^{1}$, Ryuzo Takaya ${ }^{2}$, \\ Takashi Uemura², Naoki Namba², Noriko Nariyama², Yasuhiro Kawasaki², \\ Yasuhiro Fukunaga², and Hiroshi Tamai² \\ ${ }^{1}$ Department of Pediatrics, Hirakata Municipal Hospital, Osaka, ${ }^{2}$ Department of Pediatrics, Osaka \\ Medical College, Osaka, ${ }^{3}$ Tokuda Pediatrics Clinic, Hyogo, ${ }^{4}$ Abuyama Pediatrics Clinic, Osaka, Japan
}

\begin{abstract}
We evaluated age-related insulin resistance in obese and non-obese children of different ages using the insulin sensitivity test to determine the glucose clearance (GC) calculated for the steady-state plasma glucose level (SSPG). Concomitant infusion of glucose, insulin and somatostatin should virtually eliminate endogenous glucose formation. At steady-state, glucose utilization should be equal to the infusion rate. The higher the SSPG is, the lower the insulin sensitivity is. GC calculated for SSPG reflects the insulin resistance. Thirty-one boys ( $7-17$ years) and 29 girls (5-18 years) were recruited for the study. The subjects of each sex were divided into three groups, according to the level of percentage of body fat (\%fat) (boys; $<30 \%, 30 \% \sim 40 \%,>40 \%$, girls; $<20 \%, 20 \% \sim 30 \%,>30 \%$ ). Body composition was determined using bio-electrical impedance analysis (BIA). We investigated the correlation between glucose clearance (GC) and age. In girls, an inverse correlation was found between GC and age for the group with \%fat $<20 \%$ ( $y=-$ $9.95 \mathrm{x}+190.7, \mathrm{P}=0.0073, \mathrm{r}=0.728$ ), but no correlation were found in the other groups. Also, no correlation was found in any group of boys. However GC tended to decrease as \%fat increased irrespective of the age. Both an increase of \%fat and age were important factors related to the decrease of insulin sensitivity in children. We consider that evaluation of body composition, nutrition, and lifestyle in children are important for the prevention of diseases such as syndrome X (comprising hyperinsulinemia, hypertension, and type 2 diabetes) and the deadly quartet (type 2 diabetes, obesity, hypertension, dyslipidemia, and atherosclerosis) caused by insulin resistance.
\end{abstract}

Key words: insulin resistance, insulin sensitivity, different ages, children, percentage of body fat

\section{Introduction}

Insulin resistance (IR) has been reported to occur in several diseases, such as type 2 diabetes,

Received: August 4, 2000

Accepted: January 2, 2001

Correspondence: Dr. Keisuke Okasora, Department of Pediatrics, Hirakata Municipal Hospital, 2-14 Kinyahonmachi, Hirakata, Osaka 573-1013, Japan type 1 diabetes, and obesity (1-3). Insulin resistance has attracted attention because it causes syndrome X (comprising hyperinsulinemia, hypertension, and type 2 diabetes) (4), as well the deadly quartet (type 2 diabetes, obesity, hypertension, dyslipidemia, and atherosclerosis) (5) in patients with obesity, type 2 diabetes, and type 1 diabetes. In advanced countries, the number of children with obesity and/or type 2 
diabetes has been increasing for several decades, but few studies have evaluated insulin sensitivity in children. In this study, we performed the insulin sensitivity test by glucose, insulin, and somatostatin infusion in obese and non-obese children to evaluate insulin resistance at different ages. We investigated the correlation between glucose clearance, the percentage of body fat (\%fat), and age.

\section{Subjects and Methods}

Thirty-one boys ( $7-17$ years, median age 12 years) and 29 girls (5-18 years, median age 13 years) were recruited for this study. The boys and girls were each divided into three groups according to the \%fat in roughly equal numbers (boys: $<30 \%$, $\mathrm{n}=12,30 \% \sim 40 \%, \mathrm{n}=12,>40 \%, \mathrm{n}=7$, girls: $<20 \%, \mathrm{n}=12$, $20 \% \sim 30 \%, n=11,>30 \%, n=6$ ). The subjects had varieties of conditions, such as anorexia nervosa, psychosomatic disease, and obesity. Healthy controls were also included. Bio-electrical impedance analysis (BIA) was performed to measure the body composition, especially the \%fat using an SS103 Bio-impemeter (Sekisui Ltd., Japan). We investigated the correlation between GC and age. Insulin sensitivity (IS) was estimated by a modification of the insulin sensitivity test described previously (6), with somatostatin being replaced by a somatostatin analog (SA) (Sandostatin, Novartis Pharmaceuticals Ltd., Basel, Switzerland). In brief, $40 \mu \mathrm{g}$ of SA was injected as a bolus, after which constant infusion at a rate of $3 \mathrm{ml} / \mathrm{kg} / \mathrm{hr}$ was started with $0.77 \mathrm{mU} / \mathrm{kg} /$ min of insulin, $75 \mu \mathrm{g} / \mathrm{h}$ of SA, and $6 \mathrm{mg} / \mathrm{kg} / \mathrm{min}$ of glucose in a $5 \mathrm{mEq}$ potassium solution via an antecubital vein. Blood samples were drawn at 0 , 30, 60, 90 and 120 min for determination of plasma glucose, insulin, CPR, growth hormone, and serum potassium. The steady state plasma glucose (SSPG) level was measured after infusion for 120 min, and IS was calculated by the formula reported previously (6). The subjects were admitted to hospital as in-patients for the purpose of the study.
Verbal informed consent for this study was obtained from the parents of the subjects.

\section{Statistical Analysis}

Statistical analysis was performed using the Stat-View program (version 4.0, Abacus Concepts, Inc., Berkeley, California) and a Macintosh computer. Correlations between body mass index (BMI) and GC, or \%fat and GC in all subjects, and correlations between GC and age in each group were calculated by regression analysis.

\section{Results}

In five subjects, slight nausea was noted during the test, but there were no other significant side effects. The plasma glucose level reached a steady state after $90 \mathrm{~min}$, and SSPG was determined using the plasma glucose level at 120 min. Both BMI and \%fat showed an inverse correlation with GC in boys (Fig. 1). The correlation coefficients were significant -0.714 $(\mathrm{P}<0.0001)$ and $-0.649(\mathrm{P}<0.0001)$, respectively. In girls, BMI also showed an inverse correlation with GC and the correlation coefficient was -0.562 $(\mathrm{P}<0.0001)$ (Fig. 2). However \%fat showed no significant correlation with GC in girls. As shown Fig. 3, GC decreased with age among girls with $\%$ fat $<20 \%$, and the correlation coefficient was $-0.728(\mathrm{P}=0.0073)$. There was no significant correlation between age and GC in the other groups of girls or boys. However GC tended to be lower in both sexes as \%fat increased irrespective of age (Figs. 3, and 4).

\section{Discussion}

We used BIA to measure the \%fat. Hydrodensitometry has been established and accepted as a standard for the assessment of body composition. Recently dual energy X-ray absorptiometry (DXA) has been shown to be reliable for measurement of body composition. However neither methods are suitable for clinical 

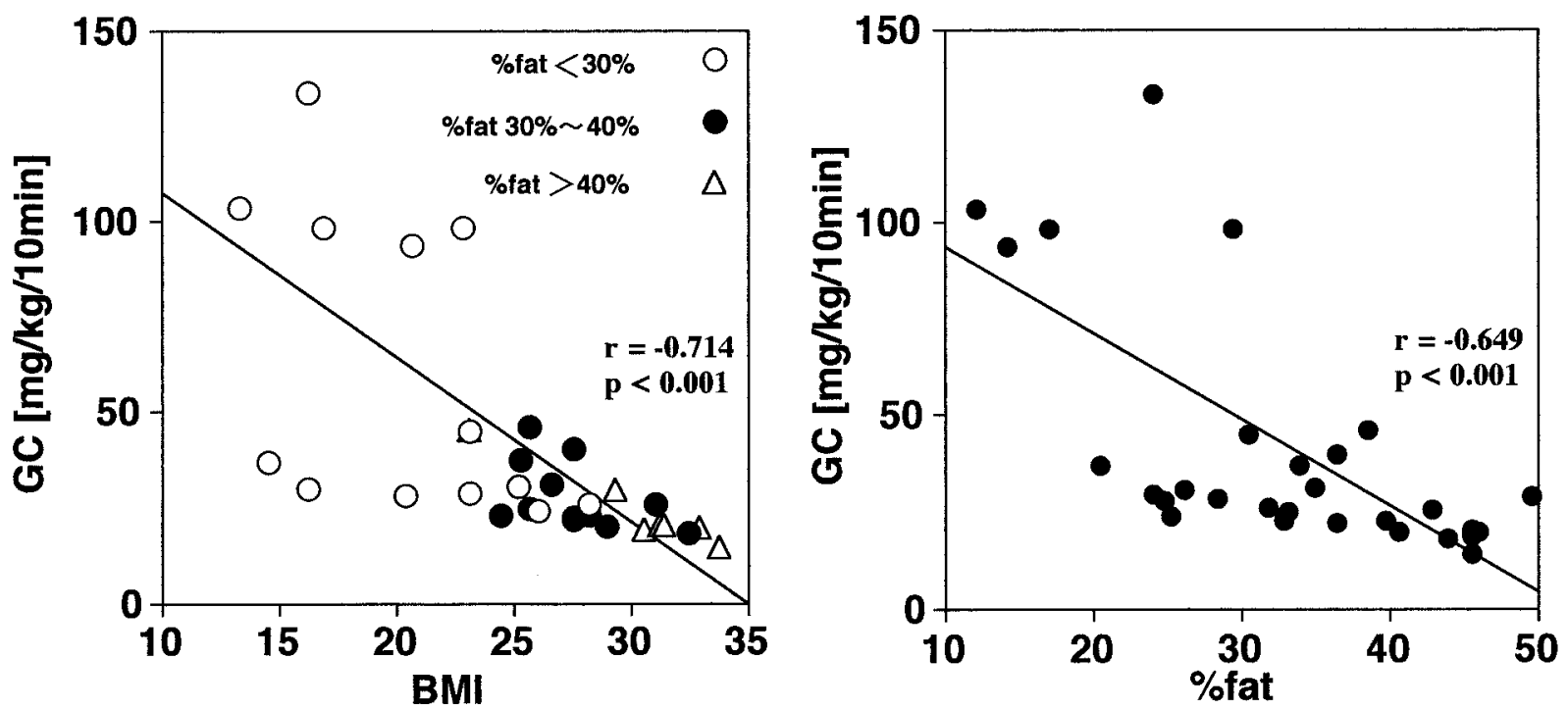

Fig. 1 Correlation between glucose clearance and BMI, and \%fat in boys. BMI and \%fat showed an inverse correlation with GC. The correlation coefficient were $-0.714(\mathrm{P}<0.0001)$ and $-0.649(\mathrm{P}<0.0001)$, respectively.
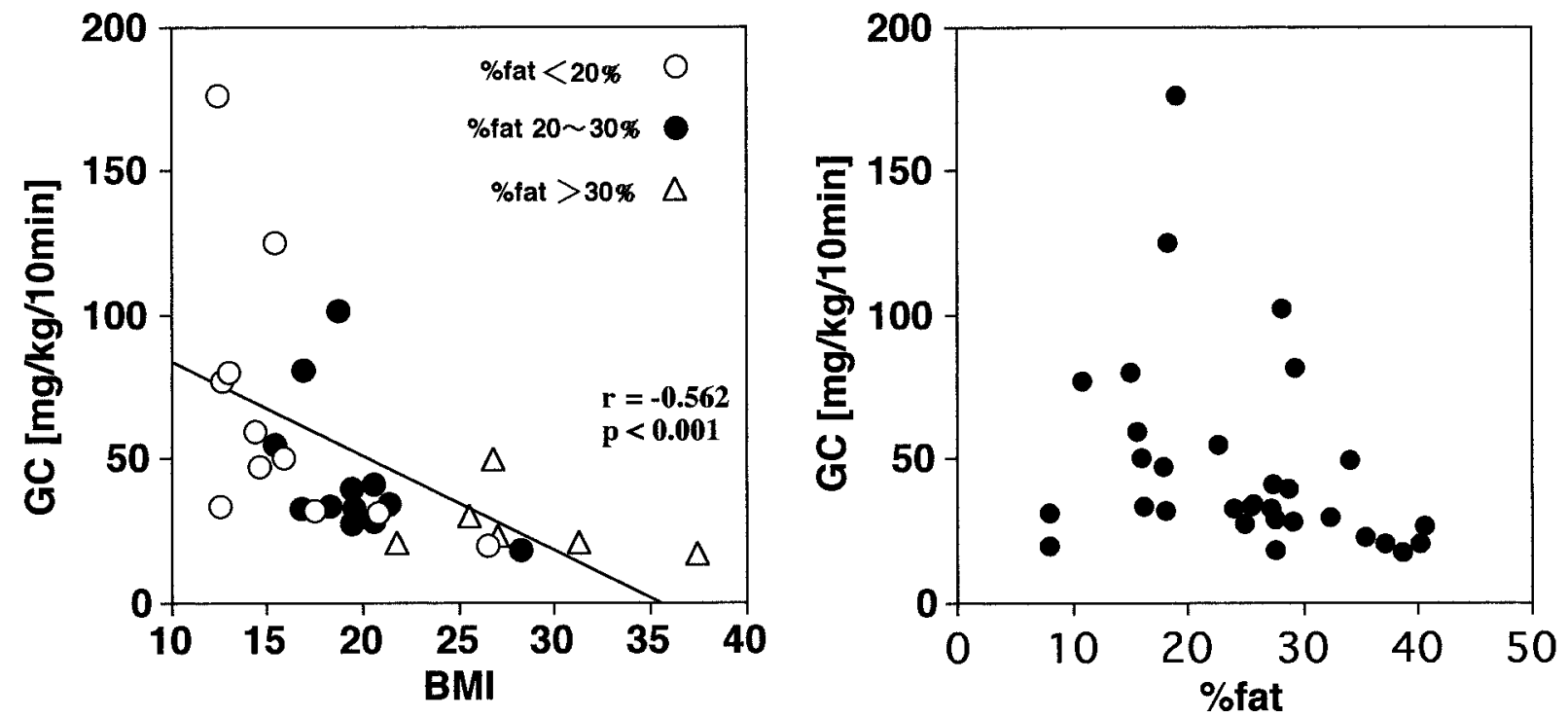

Fig. 2 Correlaton between glucose clearance and BMI and \%fat in girls. BMI showed an inverse correlation with GC, and the correlation coefficient was -0.562 ( $\mathrm{P}<0.001)$. However, \%fat showed no significant correlation with GC.

screening because of practical considerations. We have already investigated the characteristics of BIA. In the extremely underweight, \%fat was greater by BIA than by DXA, while the opposite occurred in the extremely overweight. However, as both methods were generally consistent with each other, BIA seemed to be suitable for daily clinical use because of its safety, simplicity, 


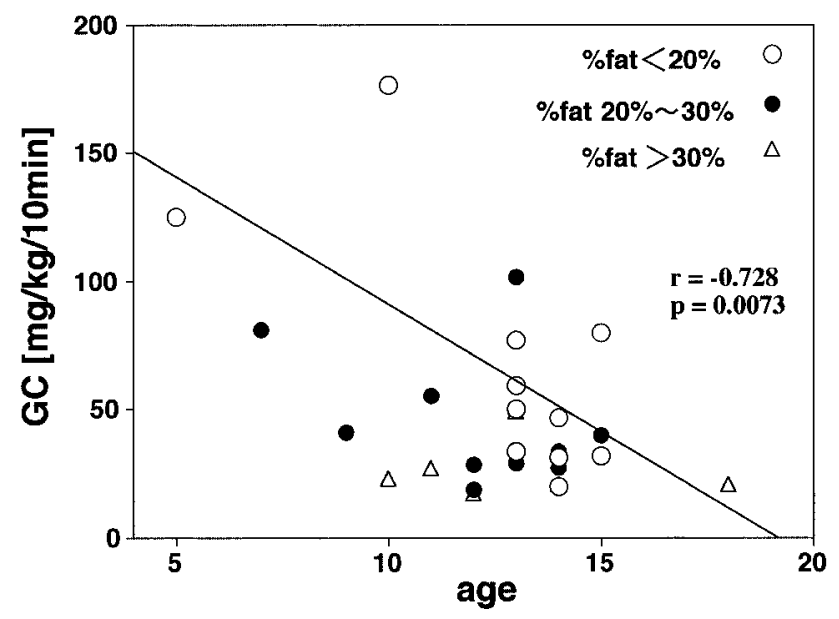

Fig. 3 Correlation between glucose clearance and age in girls. In the group with \%fat<20\%, GC decreased with age. The correlation coefficient between GC and \%fat was -0.728 $(=0.0073)$.

rapidity and low cost (7). Somatostatin has been shown to suppress endogenous secretion of insulin, glucagon, and growth hormone. This property is used to determine SSPG more accurately for estimating IS by continuous infusion of glucose, insulin and somatostatin. At steady state, glucose utilization should be equal to the infusion rate. The higher the SSPG is, the less is the IS. GC calculated for SSPG reflects the insulin resistance. When assessed on the basis of GC in children, there was a decrease of IS with increasing age and an increase of body fat. It was reported that IR is greater in obese subjects, especially those with visceral fat obesity rather than subcutaneous fat obesity (8). Recently, the secretion of TNF- $\alpha$ from adipocytes was recognized to be one of the factors involved in IR (9). TNF- $\alpha$ suppresses the expression of GLUT4 m-RNA by fat cells. Also, the blocking of signal transduction after insulin receptor stimulation seems to be another mechanism by which TNF- $\alpha$ causes IR. A relation between puberty and IR has also been described (10), with IR increasing in the early pubertal stage.

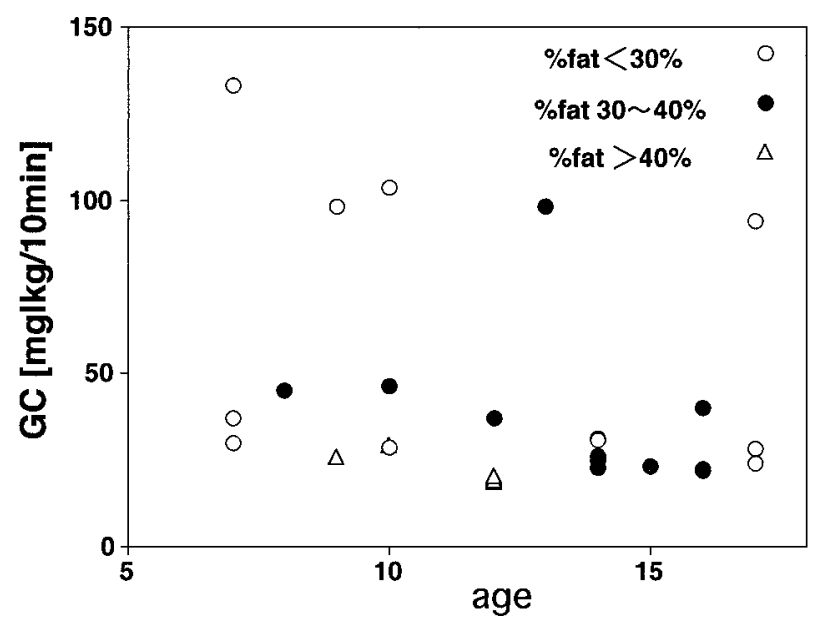

Fig. 4 Correlation between glucose clearance and age in boys. There was no significant correlation, but GC tended to decrease, as \%fat increased without relation to the age.

When the relationship between IGFBP-1 and IR was investigated (10), insulin-mediated suppression of IGFBP-1 in obese children seemed to increase free IGF-1 levels and thus contribute to somatic growth. In the present study, IS was shown to be reduced by superfluous body fat even at a young age. On the other hand, IS was variable in low fat subjects at a young age, possibly because IS was largely influenced by differences in activity if subjects are non-obese. A decrease of the action of insulin is related to the accentuation of lipolysis. Glucose uptake by skeletal muscle subsequently decreases and hepatic glyconeogenesis increases. Hyperglycemia is also related to lower glucose utilization in skeletal muscle (11).

In conclusion, obesity causes IS to decrease and predispose to disease, such as syndrome $\mathrm{X}$ (comprising hyperinsulinemia, hypertension, and type 2 diabetes) and the deadly quartet (type 2 diabetes, obesity, hypertension, dyslipidemia, and atherosclerosis) even at a young age. We consider that the evaluation of body composition, nutrition, and lifestyle in children are important for the prevention of diseases caused by IR. 


\section{References}

1. Shen SW, Reaven GM, Farquhar JW. Comparison of impedance to insulin-mediated glucose uptake in normal subjects and in subjects with latent diabetes. J Clin Invest 1970; 49: 2151-2160.

2. Rabinowitz DK, Zierler KL. Forearm metabolism in obesity and its response to intra-arterial insulin. Characterization of insulin resistance and evidence for adaptive hyperinsulinism. J Clin Invest 1962; 41: 2173-2181.

3. Ferrannini EG, Buzzigoli R, Bonadonna MA, Giorico M, et al. Insulin resistance in essential hypertension. N Engl J Med 1987; 317: 350-357.

4. Reaven GM. Role of insulin-resistance in human disease. Diabetes 1988; 37: 1595-1607.

5. DeFronzo RA, Ferrannini E. Insulin resistance: A multifaceted syndrome responsible for type 2 diabetes, obesity, hypertension, dyslipidemia, and atherosclerotic cardiovascular disease. Diabetes Care 1991; 14: 173-194.

6. Harano Y, Ohgaku S, Hidaka H, Haneda H, et al. Glucose, insulin and somatostatin infusion for the determination of insulin sensitivity. $J$ Clin
Endocrinol Metab 1977; 45: 1124-1127.

7. Okasora K, Ryuzo T, Masakuni T, Yasuhiro F, et al.. Comparisone of bioelectrical impedance analysis and dual energy X-ray absorptiometry for assessment of body composition in children. Pediatrics International 1999; 41: 121-125.

8. Yamashita S, Kotani K, Nakamura T, Takemura K, et al. Insulin resistance and body fat distribution. Diabetes Care 1996; 19: 287-291.

9. Hostamisligil GS, Arner P, Caro JF, Atkinson RL, et $a l$. Increased adipose tissue expression of tumor necrosis factor- $\alpha$ in human obesity and insulin resistance. J Clin Invest 1995; 95: 2409-2415.

10. Travers SH, Labarta JI, Gargosky SE, Rosenfeld $\mathrm{RG}$, et al. Insulin-like growth factor binding protein-I levels are strongly associated with insulin sensitivity and obesity in early pubertal children. J Clin Endocrinol Metab 1998; 83: 1935-1939.

11. Kawamori R, Morishima T, Ikeda M, Kubota M, et $a l$. Effect of strict metabolic control on glucose handing by the liver and peripheral tissue in noninsulin dependent diabetes mellitus. Diab Res Clin Pract 1994; 23: 155-161. 